\title{
A method for getting a finite $\alpha$ in the IR region from an all-order beta function
}

\author{
Zhi-Yuan Zheng ${ }^{1,2, a}{ }_{(D)}$, Gao-Liang Zhou ${ }^{3, b}$ \\ ${ }^{1}$ Key Laboratory of Theoretical Physics, Institute of Theoretical Physics, Chinese Academy of Sciences, Beijing 100190 , \\ People's Republic of China \\ ${ }^{2}$ School of Physical Sciences, University of Chinese Academy of Sciences, No.19A Yuquan Road, Beijing 100049, China \\ ${ }^{3}$ College of Science, Xi' an University of Science and Technology, Xi' an 710054, People's Republic of China
}

Received: 22 June 2017 / Accepted: 14 November 2017 / Published online: 24 November 2017

(C) The Author(s) 2017. This article is an open access publication

\begin{abstract}
The analytical method of the QCD running coupling constant is extended to a model with an all-order beta function which is inspired by the famous Novikov-ShifmanVainshtein-Zakharov beta function of $N=1$ supersymmetric gauge theories. In the approach presented here, the running coupling is determined by a transcendental equation with non-elementary integral of the running scale $\mu$. In our approach $\alpha_{\text {an }}(0)$, which reads 0.30642 , does not rely on any dimensional parameters. This is in accordance with results in the literature on the analytical method of the QCD running coupling constant. The new "analytically improved" running coupling constant is also compatible with the property of asymptotic freedom.
\end{abstract}

\section{Introduction}

The renormalization scale dependence or evolution of running coupling constant is described by the beta function of a theory concerned. The explicit expression for the beta function can be obtained by means of perturbation methods, and the validity of its use depends on whether its resultant coupling constant is excessively large or not. Solving a given beta function, we can obtain an expression for the running coupling constant, which may blow up or become sufficiently large somewhere, indicating that the perturbation breaks down and showing the invalidity of using this given beta function. This problem leads us to expect that, as in Ref. [1], the running coupling constant must cease to increase somewhere (i.e. freeze somewhere).

The freezing of the running coupling constant (FORCC) is closely related to the explicit form of the beta function. Using

\footnotetext{
a e-mail: zhengzy@itp.ac.cn

be-mail: zhougl@itp.ac.cn
}

the beta function to study the FORCC is meaningful and direct; the freezing of the running coupling constant might appear as a result of the beta function's vanishing - FORCC is related to an infrared or ultraviolet zero of the beta function. In Ref. [2] the infrared zero of the beta function was studied at three-loop order. In Refs. [3-9] this was carried out to fourloop order. Recently, in Ref. [10] this has been carried out to five-loop order.

The property of freezing coupling constant $\alpha$ at low energy scales in QCD has been studied for many years $[1,11]$ and been widely used in QCD phenomenology [11-18]. The phenomenological evidence for the running coupling constant of QCD to freeze in the IR region is numerous and increasing. Various models have been developed to investigate FORCC, and the predicted value for $\alpha_{s}(0)$ of QCD ranges from 0.4 to 1 in phenomenology $[15,16,19-22]$. Also, there are theoretical reasons in favor of FORCC [2,22]. Even though the behavior of running coupling of QCD in the IR region is nonperturbative in the usual sense, in Ref. [16] it was shown that perturbation theory provided some theoretical evidence for FORCC.

Many methods have been used to investigate FORCC. For example, in Ref. [23] the Schwinger-Dyson equation was used to investigate FORCC. Another well-known way to study FORCC is to study the coupling constant directly we can obtain the knowledge of the running coupling in the IR region from the knowledge of it in the UV region [1]. In Ref. [1], through the use of an "analytization procedure", an "analytically improved" expression for the running coupling was obtained, which is free of a ghost pole and has a universal limit value. The "analytization procedure" elaborated in Refs. [24-26] and used in Ref. [1] includes three steps:

1. Finding an explicit expression for $\alpha_{s}\left(\mu^{2}\right)$ in the Euclidean region. 
2. Performing analytical continuation into the Minkowski region. Extracting its imaginary part for defining the spectral density by $\rho_{\mathrm{RG}}(\sigma, \alpha)=\operatorname{Im} \alpha(-\sigma-i \varepsilon, \alpha)$.

3. Using $\rho_{\mathrm{RG}}$ to define an analytically improved running coupling constant in the Euclidean region.

In a sense these three steps are perturbative, since $\alpha$ is obtained by perturbation methods usually. An all-order beta function was first proposed in Ref. [27]. A proof of this is given in Ref. [28]. In our work, this beta function is used to extract $\alpha_{\text {an }}(\mu)$ in a similar way to the way used in Ref. [1].

The remainder of this paper is organized as follows. In Sect. 2, we start with an all-order beta function and concentrate on eliminating the ghost pole. In Sect. 3, after having removed the singularity, we get an equation from which we define a new analytically improved running coupling which is free of a ghost pole and does respect asymptotic freedom. In Sect. 4, the issue of the scheme dependence is discussed.

\section{Elimination of singularity}

As has been said before, an all-order beta function was first proposed in Ref. [27] and proved in Ref. [28], which takes the form

$$
\frac{\beta(\alpha)}{\alpha}=-\frac{\alpha}{2 \pi} \frac{\alpha+\sum_{r=1}^{p} \alpha_{r} N_{r} \gamma_{r}}{1-\frac{\alpha}{2 \pi} \alpha_{g}} .
$$

For pure Yang-Mills theory this can be simplified to

$$
\frac{\beta_{\mathrm{YM}}(\alpha)}{\alpha}=-\frac{11}{3} \frac{\alpha}{2 \pi} \frac{C_{2}[G]}{1-\frac{\alpha}{2 \pi} \frac{17}{11} C_{2}[G]} .
$$

In $\mathrm{SU}(N)$ gauge theory, $C_{2}(G)$ is just $N$. Carrying out the obvious transformation $\frac{\alpha}{2 \pi} \rightarrow \alpha$ converts Eq. (2) to a new compact beta function in the form

$\beta_{\mathrm{YM}}(\alpha)=-\frac{C_{1} \alpha^{2}}{1-C_{2} \alpha}$,

where $C_{1}=11 C_{2}(G) / 3$ and $C_{2}=17 C_{2}(G) / 11$. The general solution of this equation is of the form

$C_{2} \ln \frac{\alpha(\mu)}{\alpha(\Lambda)}+\left(\frac{1}{\alpha(\mu)}-\frac{1}{\alpha(\Lambda)}\right)=C_{1} \ln \frac{\mu}{\Lambda}$,

where $\Lambda$ is just an integral constant. For later convenience, inverting Eq. (4) and making the replacement $\ln \frac{\mu}{\Lambda} \rightarrow$ $\frac{1}{2} \ln \frac{\mu_{E}^{2}}{\Lambda_{E}^{2}}$ (here $\mu_{E}^{2}=-\mu^{2}$ and $\Lambda_{E}^{2}=-\Lambda^{2}$ ), we have

$\frac{1}{C_{2} \ln \frac{\alpha(\mu)}{\alpha(\Lambda)}+\left(\frac{1}{\alpha(\mu)}-\frac{1}{\alpha(\Lambda)}\right)}=\frac{2}{C_{1} \ln \frac{\mu_{E}^{2}}{\Lambda_{E}^{2}}}$.
For simplicity, we denote by $y_{0}\left(\mu_{E}^{2}\right)$ the right-hand side of Eq. (5) and by $y_{0}(\alpha(\mu))$ the left-hand side of Eq. (5).

The analyticity property of $y_{0}(\alpha(\mu))$ is almost the same as that of the running coupling $\alpha(\mu)$ with the exception that the coupling constant is non-positive or may be a pole of $y_{0}(\alpha(\mu))$. Therefore, in this work we shall concentrate on studying the analyticity properties of $y_{0}(\alpha(\mu))$ and $y_{0}\left(\mu_{E}^{2}\right)$. For simplicity, we set $\alpha(\Lambda)=1$ and $\lambda=C_{2}$; then

$y_{0}(\alpha(\mu))=\frac{1}{\frac{1}{\alpha(\mu)}+(\lambda \ln \alpha(\mu)-1)}$.

It may be shown that the denominator of $y_{0}(\alpha(\mu))$ can be zero only if the running coupling $\alpha$ is a real number.

For the continuation of this work, we make some reasonable assumptions here; self-consistency of some of them can be verified by a later check.

Let us make the first assumption: for the running coupling to be real-valued, it is necessary that $\mu^{2}$ must be real-valued. Self-consistence of this assumption can be checked later.

The second assumption is the absence of a Landau pole. Therefore, according to asymptotic freedom, which will be discussed later, there is no point on the real axis at which $\alpha_{s}$ is negative. When $\mu_{E}^{2}$ goes to $-\infty$, according to asymptotic freedom, $\alpha(\mu)$ tends to 0 , which leads to the denominator of $y_{0}(\alpha(\mu))$ having a non-zero value. Therefore, the distance between points at which the denominator of $y_{0}(\alpha(\mu))$ vanishes and the origin must reach its maximum (if it exists) at a point on the negative real axis - we set it equal to $-\mu_{0}^{2}$.

As is well known, if a function is analytical in a connected region surrounded by a contour, we can use the Cauchy theorem

$y\left(\mu^{2}\right)=\frac{-i}{2 \pi} \oint \frac{y(x)}{x-\mu^{2}} \mathrm{~d} x$,

which in this paper is used to subtract singularity terms.

The third assumption is that the integral along an infinite contour is summed to zero. Note that $y_{0}(\alpha(\mu))$ is analytical except when $\mu_{E}^{2}$ is a pole on the real axis. Therefore, in this paper, the integral is taken over the contour shown in Fig. 1. Thus we immediately arrive at

$y_{0}\left(\mu_{E}^{2}\right)=\frac{1}{\pi} \int_{-\mu_{0}^{2}}^{\infty} \frac{\operatorname{Im} y_{0}(x+i \varepsilon)}{x-\mu_{E}^{2}} \mathrm{~d} x$.

We note that

$\operatorname{Im} \ln \frac{a+i \varepsilon}{b}= \begin{cases}0, & \text { if } a<0 \text { and } b<0 ; \\ -\pi, & \text { if } a>0 \text { and } b<0 .\end{cases}$

Thus Eq. (8) can be further simplified to

$y_{0}\left(\mu_{E}^{2}\right)=\frac{2}{C_{1}} \int_{0}^{\infty} \frac{1}{x-\mu_{E}^{2}} \frac{\mathrm{d} x}{\ln ^{2}\left|\frac{x}{\Lambda_{E}^{2}}\right|+\pi^{2}}$. 


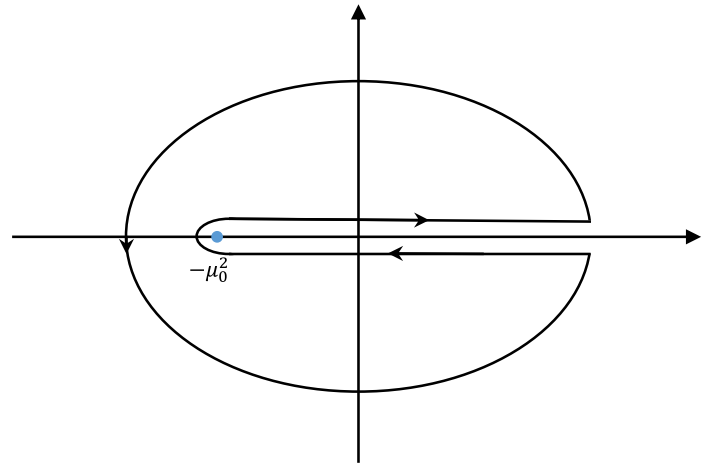

Fig. 1 Cauchy theorem: The horizontal axis represents the real part of $\mu_{E}^{2}$, and the vertical axis represents the imaginary part of $\mu_{E}^{2}$

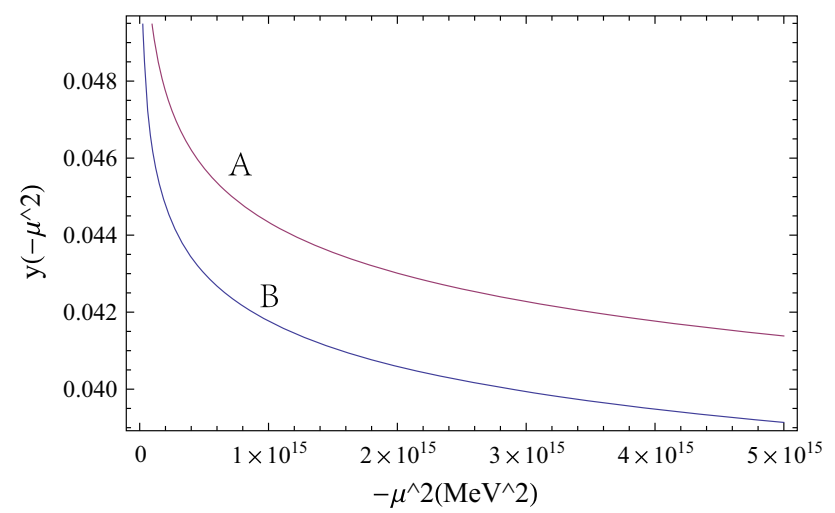

Fig. 2 The curves $\mathrm{A}$ and $\mathrm{B}$ are $y_{\mathrm{an}}\left(\mu^{2}\right)$ for $\Lambda=400 \mathrm{MeV}$ and $\Lambda=$ $200 \mathrm{MeV}$, respectively

\section{Evaluation of coupling constant}

It is almost always the case that the values of $y_{0}\left(\mu_{E}^{2}\right)$ are related to $\Lambda$. The two cases $\Lambda=200 \mathrm{MeV}$ and $\Lambda=$ $400 \mathrm{MeV}$ are shown in Fig. 2. However, there is the one obvious exception that $y_{\mathrm{an}}(0)$ is independent of the value of $\Lambda$. Therefore, the value for $\alpha_{\mathrm{an}}(0)$ is independent of $\Lambda$.

Since the first part of the integrand in Eq. (10) is a monotone decreasing function of energy scale $\mu$, it is obvious that, as can be seen from Fig. $2, y_{0}\left(\mu_{E}^{2}\right)$ is a monotone decreasing function of the energy scale.

By straightforward calculation, we obtain an explicit expression for Eq. (10) in the form

$y_{\mathrm{an}}(\mu)=\frac{2}{C_{1}}\left(\frac{1}{\ln \frac{\mu^{2}}{\Lambda^{2}}}+\frac{\Lambda^{2}}{\Lambda^{2}-\mu^{2}}\right)$,

which decreases monotonically from $2 / C_{1}$ to zero as $\mu$ varies from zero to infinity, and which is free of a ghost pole, to be compared with the original expression $2 /\left(C_{1} \ln \mu^{2} / \Lambda^{2}\right)$.

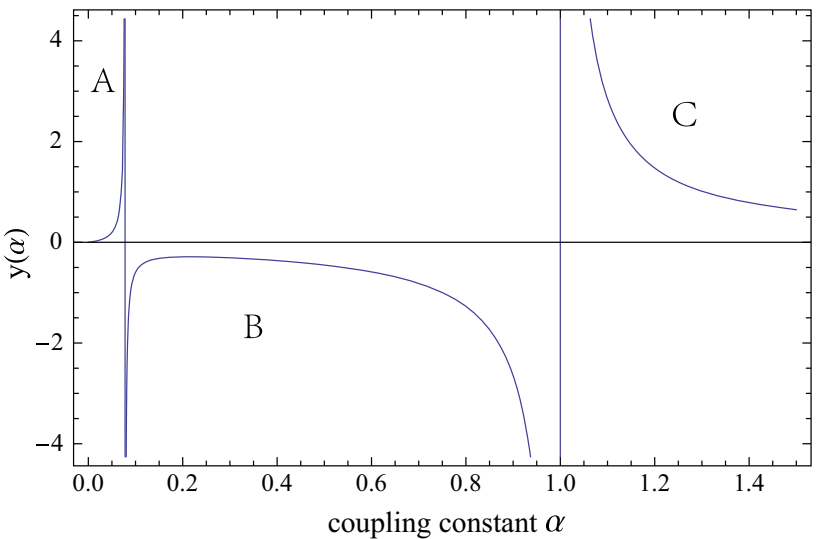

Fig. 3 The horizontal axis represents the coupling constant $\alpha_{\mathrm{an}}$, the vertical axis represents $y_{\mathrm{an}}\left(\alpha_{\mathrm{an}}\right)$. Region B has no corresponding $y_{\mathrm{an}}(\mu)$, and it should not be taken into consideration later

The value of $\alpha_{\mathrm{an}}\left(\mu^{2}\right)$, in our approach, is determined by the equation

$$
\begin{aligned}
y_{\mathrm{an}}\left(\alpha_{\mathrm{an}}(\mu)\right) & =\frac{\alpha_{\mathrm{an}}(\mu)}{\left[1+\alpha_{\mathrm{an}}(\mu)\left(\lambda \ln \alpha_{\mathrm{an}}(\mu)-1\right)\right]} \\
& =\frac{2}{C_{1}}\left(\frac{1}{\ln \frac{\mu^{2}}{\Lambda^{2}}}+\frac{\Lambda^{2}}{\Lambda^{2}-\mu^{2}}\right),
\end{aligned}
$$

which is an analytically improved equation corresponding to $y_{0}(\alpha(\mu))=y_{0}\left(\mu_{E}^{2}\right)$.

As can be seen from Fig. 3 the new analytically improved running coupling $\alpha_{\text {an }}$ as a function of $\mu$ is two-valued: each value of the $y_{\mathrm{an}}(\mu)$ corresponds to two different values of $\alpha_{\mathrm{an}}$. The difference between these two choices of value for the running coupling, as can be seen from Fig. 3, is obviously large and varies monotonously with the energy scale. Therefore, the determination of the running coupling is a little more complicated.

The property of $y_{\mathrm{an}}\left(\alpha_{\mathrm{an}}(\mu)\right)$ as a function of $\alpha_{\mathrm{an}}(\mu)$ is somewhat complicated. Differentiating $y_{\mathrm{an}}\left(\alpha_{\mathrm{an}}\right)$ with respect to $\alpha_{\mathrm{an}}$, we have

$y_{a n}^{\prime}\left(\alpha_{\mathrm{an}}(\mu)\right)=\frac{1-\lambda \alpha_{\mathrm{an}}(\mu)}{\left(1+\alpha_{\mathrm{an}}(\mu)\left(\lambda \ln \alpha_{\mathrm{an}}(\mu)-1\right)\right)^{2}}$,

whose numerator is $1-\lambda \alpha_{\mathrm{an}}(\mu)$ and whose denominator is always nonnegative. We note that, as long as $\alpha_{\text {an }}(\mu)$ does not exceed $\frac{1}{\lambda}, y_{\mathrm{an}}^{\prime}\left(\alpha_{\mathrm{an}}(\mu)\right)$ maintain its positivity. The appearance of abrupt changes shown in Fig. 3 results from the vanishing of the denominator of $y_{\mathrm{an}}^{\prime}\left(\alpha_{\mathrm{an}}(\mu)\right)$.

It may be shown that, of the three regions represented by A, B and C, respectively, in Fig. 3, the middle one (represented by B) corresponds to an un-physical region and therefore can be discarded, since $y_{\mathrm{an}}(\mu)$, with the explicit expression shown in Eq. (11), is always positive. Since $y_{\mathrm{an}}(\mu)$ is 
a monotone decreasing function of the energy scale, of the remainder region $\mathrm{A}$ and $\mathrm{C}$, the left region $\mathrm{A}$ in Fig. 3 corresponds to a region obeying asymptotic freedom (as the energy scale increases, $y_{\mathrm{an}}(\mu)$ decreases, which leads to the decrement of $\alpha_{\text {an }}(\mu)$ ), and the right region $\mathrm{C}$ in Fig. 3 corresponds to a region violating asymptotic freedom (as the energy scale increases, the coupling constant increases, and, what is worse, in the UV region the coupling constant blows up). Taking our assumption of asymptotic freedom, physical intuition and mathematical continuity into consideration, region $\mathrm{C}$ also can be discarded. The distinction between regions $\mathrm{A}$ and $\mathrm{C}$ also may be traced back to the explicit form of the all-order beta function in Eq. (3). As the energy scale changes from the IR region to the UV region, evolution of the coupling constant is essentially determined by the initial value of the running coupling in the IR region. If this value is smaller than $\frac{1}{C_{2}}$, asymptotic freedom is obeyed, otherwise it is violated dramatically.

Now, for fixed $\mu$, taking all the above discussion into consideration, we can fix the corresponding $\alpha_{a n}(\mu)$. Even though it is still impossible to acquire an expression for $\alpha_{\mathrm{an}}(\mu)$ in terms of $\mu$ explicitly, the numerical value for $\alpha_{\text {an }}$ can be obtained for any $\mu$. Letting $\mu$ vanish, for SU(3) gauge theory, we get a numerical value 0.0487688 in region A of Fig. 3 (the region obeying asymptotic freedom) and a numerical value 3.8413 in region C of Fig. 3 (the region violating asymptotic freedom dramatically). Therefore, we choose 0.0487688 as our result. Since the transformation $\frac{\alpha}{2 \pi} \rightarrow \alpha$ has been made earlier, the running coupling constant in the IR region freezes at 0.30642 .

\section{Discussion of scheme dependence}

As is well known, for a given theory to be renormalizable, we need impose the requirement that the divergent part of any one-particle irreducible diagram can be cancelled by a local counterterm generated from the bare Lagrangian of this given theory. Therefore, once the divergences of a given theory have been subtracted out by a certain renormalization scheme, one is still free to perform a further finite renormalization, thus resulting in the may-be scheme dependence of quantities in field theory. In this section, our discussion is restricted to the class of mass-independent renormalization schemes.

As far as we are concerned here, for the beta function usually only the first two non-vanishing coefficients of the expansion for the beta function are scheme independent. There is one obvious may-be exception to this statement just given above. If, for a given theory, an all-order beta function can be obtained in a certain renormalization scheme and written in the form $\beta(\alpha)=\frac{\beta_{1} \alpha^{n}}{1-\frac{\beta_{2}}{\beta_{1}} \alpha^{m}}$,

and if this form can be maintained in anther scheme, it is obvious that the all-order beta functions in these two schemes are equal to each other.

The expression for the beta function expanded in terms of the running coupling is usually of the form

$\beta(\alpha)=\frac{\mathrm{d} \alpha}{\mathrm{d} \ln \mu}=\beta_{0} \alpha^{n_{0}}+\beta_{1} \alpha^{n_{1}}+\beta_{2} \alpha^{n_{2}}+\cdots$,

which can be rewritten as

$\beta(\alpha)=\frac{\mathrm{d} \alpha}{\mathrm{d} \ln \mu}=\frac{\beta_{0} \alpha^{n_{0}}}{1+\beta_{1}^{\prime} \alpha^{n_{1}^{\prime}}+\beta_{2}^{\prime} \alpha^{n_{2}^{\prime}}+\cdots}$.

The new coefficients of Eq. (16) can be obtained by matching with Eq. (15) order by order.

By the same procedures as carried out above, we may obtain an equation between the new analytically improved coupling $\alpha_{\text {an }}$ and energy scales of the form

$$
\begin{gathered}
\frac{1}{a_{0} \ln \alpha_{\mathrm{an}}(\mu)+\sum_{i \neq 0} a_{i} \alpha_{\mathrm{an}}(\mu)^{i}+C} \\
=2\left(\frac{1}{\ln \frac{\mu^{2}}{\Lambda^{2}}}+\frac{\Lambda^{2}}{\Lambda^{2}-\mu^{2}}\right),
\end{gathered}
$$

where $\Lambda$ is just an integral constant and $\mathrm{C}$ also a constant. It is obvious that if $\alpha_{\mathrm{an}}$ is in the perturbation region (i.e. small enough), of all the terms in the denominator of the expression in the left-hand side of Eq. (17), the term with a lower index $i$ contributes more (the first two non-vanishing terms with scheme independent coefficients contribute most).

To conclude this section, we may make the following remarks concerning the scheme dependence of the new "analytically improved" freezing coupling. First, in our approach the existence of the freezing coupling is independent of the renormalization scheme we choose, though the explicit value is scheme dependent. Second, if the running coupling is in the perturbation region, the freezing coupling is effectively (within the accuracy required) determined by a finite number of non-vanishing coefficients of the beta function.

\section{Conclusion}

The appearance of a ghost pole in the expression for the running coupling of QCD or QED leads to the hypothesis that the running coupling must freeze somewhere. In this article, starting with an all-order beta function, we have obtained an 
'analytically improved" coupling by some analyticity procedure to remove the singularity. This new running coupling is free of a ghost pole and does respect asymptotic freedom.

Much work has been done to investigate FORCC theoretically or phenomenologically. It is clear that much more work has to be done in order to get a profound understanding of this amazing property which is essential to physics.

Acknowledgements We thank Prof. Y. Q. Chen and Dr. P. Wu for helpful discussions and important suggestions on the manuscript. One of us (Z. Y. Zheng) thanks Y. Z. Xu and Z. L. Cui for giving necessary help to finish this work. The work of Z. Y. Zheng is supported by The National Nature Science Foundation of China under Grant No. 11275242. The work of G. L. Zhou is supported by The National Nature Science Foundation of China under Grant No. 11647022.

Open Access This article is distributed under the terms of the Creative Commons Attribution 4.0 International License (http://creativecomm ons.org/licenses/by/4.0/), which permits unrestricted use, distribution, and reproduction in any medium, provided you give appropriate credit to the original author(s) and the source, provide a link to the Creative Commons license, and indicate if changes were made.

Funded by SCOAP 3 .

\section{Appendix A}

In this appendix we shall prove a statement given above, and check an assumption made above.

Firstly, we prove the statement that the denominator of $y_{0}(\alpha(\mu))$ shown in Eq. (6) can be zero only if $\alpha(\mu)$ is real number.

Let us set $\alpha=r e^{i \theta}$. Then the condition for the denominator of $y_{0}(\alpha(\mu))$ to vanish can be expressed as

$$
\begin{aligned}
\lambda \theta & =\frac{1}{r} \sin (\theta), \\
1 & =\lambda \ln r+\frac{\cos (\theta)}{r} .
\end{aligned}
$$

From condition (A.1), we have

$\lambda r \leq 1$

which, noting that, for $\mathrm{SU}(3), \lambda=51 / 11$, leads to

$$
\cos (\theta)>0 \text {. }
$$

Now we can set $-\frac{\pi}{2} \leq \theta \leq \frac{\pi}{2}$. From this condition we have

$$
\begin{aligned}
& \frac{2}{\pi} \leq \lambda r \leq 1, \\
& \lambda \ln r+\frac{1}{r} \geq 1 .
\end{aligned}
$$

Note that under the condition $\frac{\pi}{2} \leq \lambda r \leq 1$ there is no solution to $\lambda \ln r+\frac{1}{r}>1-$ these two conditions contradict. Thus this statement is proved.

Secondly, we check the assumption: when $\mu^{2}$ is not real, the coupling is not real.

From Eq. 10, we can extract the imaginary part of $y_{\mathrm{an}}\left(\mu^{2}\right)$, which can be written as

$$
\begin{aligned}
& \operatorname{Im} y_{\mathrm{an}}\left(\mu_{E}^{2}\right) \\
& =\frac{2}{C_{1}} \int_{0}^{\infty} \frac{\operatorname{Im}\left(\mu_{E}^{2}\right)}{\left(x-\operatorname{Re}\left(\mu_{E}^{2}\right)\right)^{2}+\left(\operatorname{Im}\left(\mu_{E}^{2}\right)\right)^{2}} \frac{\mathrm{d} x}{\ln ^{2}\left|\frac{x}{\Lambda^{2}}\right|+\pi^{2}} .
\end{aligned}
$$

It is obvious that $\operatorname{Im}\left(\mu_{E}^{2}\right) \neq 0$ implies $\operatorname{Im} y\left(\mu_{E}^{2}\right) \neq 0$, which leads to the appearance of an imaginary part in $y_{\mathrm{an}}\left(\alpha_{\mathrm{an}}\right)$. This can happen only when $\alpha$ is not real number. Thus we finish our check.

\section{References}

1. D.V. Shirkov, I.L. Solovtsov, Phys. Rev. Lett. 79, 1209 (1997)

2. T. Banks, A. Zaks, Nucl. Phys. B 196, 189 (1982)

3. E. Gardi, M. Karliner, Nucl. Phys. B 529, 383 (1998)

4. E. Gardi, G. Grunberg, JHEP 9903, 024 (1999)

5. F.A. Chishtie, V. Elias, V.A. Miransky, T.G. Steele, Prog. Theor. Phys. 104, 603 (2000)

6. T.A. Ryttov, R. Shrock, Phys. Rev. D 83, 056011 (2011)

7. R. Shrock, Phys. Rev. D 87, 116007 (2013)

8. R. Shrock, Phys. Rev. D 87(10), 105005 (2013)

9. C. Pica, F. Sannino, Phys. Rev. D 83, 035013 (2011)

10. T.A. Ryttov, R. Shrock, Phys. Rev. D 94(10), 105015 (2016)

11. A.M. Badalian, Y.A. Simonov, Phys. Atom. Nucl. 60, 630 (1997). [Yad. Fiz. 60, 714 (1997)]

12. E. Eichten, K. Gottfried, T. Kinoshita, J.B. Kogut, K.D. Lane, T.M. Yan, Phys. Rev. Lett. 34, 369 (1975). (Erratum: [Phys. Rev. Lett. 36, 1276 (1976)])

13. E. Eichten, K. Gottfried, T. Kinoshita, K.D. Lane, T.M. Yan, Phys. Rev. D 21, 203 (1980)

14. J.L. Richardson, Phys. Lett. 82B, 272 (1979)

15. S. Godfrey, N. Isgur, Phys. Rev. D 32, 189 (1985)

16. A.C. Mattingly, P.M. Stevenson, Phys. Rev. D 49, 437 (1994)

17. Y.L. Dokshitzer, V.A. Khoze, S.I. Troian, Phys. Rev. D 53, 89 (1996)

18. A.M. Badalian, D.S. Kuzmenko, Phys. Rev. D 65, 016004 (2001)

19. A.C. Mattingly, P.M. Stevenson, Phys. Rev. Lett. 69, 1320 (1992)

20. Y.L. Dokshitzer, B.R. Webber, Phys. Lett. B 352, 451 (1995)

21. Y.L. Dokshitzer, G. Marchesini, B.R. Webber, Nucl. Phys. B 469, $93(1996)$

22. A.C. Aguilar, A. Mihara, A.A. Natale, Phys. Rev. D 65, 054011 (2002)

23. C.D. Roberts, A.G. Williams, Prog. Part. Nucl. Phys. 33, 477 (1994)

24. P.J. Redmond, Phys. Rev. 112(4), 1404 (1958)

25. P.J. Redmond, J.L. Uretsky, Phys. Rev. Lett. 1, 147 (1958)

26. N.N. Bogoliubov, A.A. Logunov, D.V. Shirkov, Sov. Phys. JETP 37(10), 574 (1960)

27. T.A. Ryttov, F. Sannino, Phys. Rev. D 78, 065001 (2008)

28. C. Pica, F. Sannino, Phys. Rev. D 83, 116001 (2011) 